Original article

\title{
Risk factors for hospital readmission of elderly patients
}

\author{
Carlotta Franchi ${ }^{\text {a, } *}$, Alessandro Nobili ${ }^{\text {a }}$, Daniela Mari ${ }^{\mathrm{b}}$, Mauro Tettamanti ${ }^{\mathrm{a}}$, Codjo D. Djade ${ }^{\mathrm{a}}$, Luca Pasina ${ }^{\mathrm{a}}$, \\ Francesco Salerno ${ }^{c}$, Salvatore Corrao ${ }^{\mathrm{d}}$, Alessandra Marengoni ${ }^{\mathrm{e}}$, Alfonso Iorio ${ }^{\mathrm{f}}$, \\ Maura Marcucci ${ }^{\mathrm{f}}$, Pier Mannuccio Mannucci ${ }^{\mathrm{g}}$ \\ and on behalf of REPOSI Investigators ${ }^{1}$
}

${ }^{a}$ Mario Negri Institute for Pharmacological Research, Milan, Italy

b Dipartimento di Scienze Cliniche e di Comunità, IRCCS Maggiore Hospital Foundation, Milan, Italy

c Internal Medicine I, Policlinico IRCCS San Donato, University of Milan, Italy

d Dipartimento Biomedico di Medicina Interna e Specialistica, University of Palermo, Italy

e Geriatric Unit, Spedali Civili, Department of Medical and Surgery Sciences, University of Brescia, Italy

${ }^{\mathrm{f}}$ Department of Internal Medicine, University of Perugia, Italy

g Scientific Direction, IRCCS Maggiore Hospital Foundation, Milan, Italy

\section{A R T I C L E I N F O}

\section{Article history:}

Received 11 July 2012

Received in revised form 2 October 2012

Accepted 19 October 2012

Available online $\mathrm{xxxx}$

\section{Keywords:}

Hospital readmission

Internal medicine and geriatric wards

Risk factors

Elderly

\begin{abstract}
A B S T R A C T
Background: The aim of this study was to identify which factors were associated with a risk of hospital readmission within 3 months after discharge of a sample of elderly patients admitted to internal medicine and geriatric wards.

Methods: Of the 1178 patients aged 65 years or more and discharged from one of the 66 wards of the 'Registry Politerapie SIMI (REPOSI)' during 2010, 766 were followed up by phone interview 3 months after discharge and were included in this analysis. Univariate and multivariate logistic regression models were used to evaluate the association of several variables with rehospitalization within 3 months from discharge.

Results: Nineteen percent of patients were readmitted at least once within 3 months after discharge. By univariate analysis in-hospital clinical adverse events (AEs), a previous hospital admission, number of diagnoses and drugs, comorbidity and severity index (according to Cumulative Illness Rating Scale-CIRS), vascular and liver diseases with a level of impairment at discharge of 3 or more at CIRS were significantly associated with risk of readmission. Multivariate logistic regression analysis showed that only AEs during hospitalization, previous hospital admission, and vascular and liver diseases were significantly associated with the likelihood of readmission.

Conclusions: The results demonstrate the need for increased medical attention towards elderly patients discharged from hospital with characteristics such as AEs during the hospitalization, previous admission, vascular and liver diseases.
\end{abstract}

(c) 2012 European Federation of Internal Medicine. Published by Elsevier B.V. All rights reserved.

\section{Introduction}

Hospital readmission within a short time from discharge is a common occurrence in the daily practice of internal medicine wards [1,2]. Some studies documented that the decrease in the rate of readmission is important in order to improve quality of care and reduce costs [3,4]. Different rates of readmissions have been found between hospitals and geographical areas $[5,6]$ and several risk factors and combinations thereof have been proposed in order to identify

\footnotetext{
* Corresponding author at: Laboratory for Quality Assessment of Geriatric Therapies and Services, Istituto di Ricerche Farmacologiche Mario Negri, Via La Masa 19, 20156 Milano, Italy. Tel.: + 3902 39014580; fax: + 390239001916 .

E-mail address: carlotta.franchi@marionegri.it (C. Franchi).

1 REPOSI denotes Registry of Polytherapies SIMI (Società Italiana di Medicina Interna).
}

patients at risk to be rehospitalized [7-9]. In the last few years, due to cost containment physicians are often prompted to minimize the length of hospital stay. Shortening of hospitalization length is a factor often associated to a higher risk of readmission [10], with the paradoxical effects of decreasing the quality of care and increasing secondary expenses due to the incomplete patient recovery. For this reason it has been suggested that high and rising readmission rates may be "the price of early discharge" [11]. Older people are at increased risk of hospital readmissions, so that a number of studies have chosen to study the risk factors of hospital readmission in the elderly [9]. For instance, Silverstein et al. conducted a retrospective cohort study of people aged 65 or older using administrative data of seven acute care hospitals in order to develop predictors of 30-day readmission [7], but fewer studies focused their attention on predictors of later hospital readmission in the elderly [12]. The aim of this study was to analyze which factors are associated with an increased 
risk of readmissions within 3 months of the index date of discharge of a cohort of 65 years or older patients evaluated in the frame of the network of Italian internal medicine and geriatric wards participating in 2010 in the Registro Politerapie SIMI (REPOSI).

\section{Methods}

\subsection{Study setting, design and patient population}

This study was conducted in 66 hospital wards representative of the Italian internal and geriatric medicine wards, participating in the 'Registro Politerapie SIMI' (REPOSI). The REPOSI is a collaborative and independent study of the Italian Society of Internal Medicine (SIMI) and the Mario Negri Institute of Pharmacological Research. The design was described in details elsewhere [13]. In brief, patients aged 65 years or older consecutively admitted to hospital during four periods that lasted four weeks and separated each from the other for three months, were enrolled in the study. A standardized web-based case report form was filled in by the attending physicians, including socio-demographic factors, clinical parameters, diagnoses and medications prescribed at both hospital admission and discharge, adverse events (AEs) during hospitalization, comorbidity according to the Cumulative Illness Rating Scale (CIRS) [14], performance in basic activities of daily living according to Barthel index scale [15], cognitive status according to the Short Blessed Test [16] and presence of depression using Geriatric Depression Scale [17]. Follow-up, addressed to the patient or his relative and performed 3 months after hospital discharge by physician telephone interview, collected data on mortality, new diagnoses, new hospital admission, drug regimen, AEs and Barthel index. All the collected data were checked by a central monitoring institution (the Mario Negri Institute for Pharmacological Research, Milan). The study was approved by the Ethical Committee of the IRCCS Cà Granda Maggiore Policlinico Hospital Foundation, Milan.

\subsection{Outcomes}

The primary outcome of this study was patient hospital readmission within 3 months after discharge from the first admission (index hospitalization). Rehospitalization rate was calculated only for patients whose case report form was filled in the section relative to the 3 months follow-up. The variables analyzed were: demographic data (age, sex, education, marital status, body mass index, and lifestyle habits), clinical variables (length of stay and previous hospital admission in the 6 months before the index admission, number of diagnoses and drugs at discharge, cognitive status [Short Blessed Test-SBT], depression status [Geriatric Depression scale-GDS]), and functional variables such as ability in basic activities of daily living [Barthel index]). Comorbidity and severity indexes, according to the Cumulative Illness Rating Scale (CIRS), were calculated [14]. CIRS comorbidity index was computed by counting the number of items for which moderate to severe illness was reported (scores of 3,4 or 5), while overall illness severity was represented by the mean of the 13 CIRS items [14]. The effect of the most common diseases at discharge on readmission was also analyzed. A long length of hospital stay was defined as a stay longer than the 90th percentile of the distribution of all patients' hospital stays [12]. To study the association between AEs and readmission, the diagnoses were grouped according to the chapters of International Classification of Disease, Ninth Revision (ICD-9).

\subsection{Statistical analysis}

Association between rehospitalization and socio-demographic variables, clinical characteristics and diseases at discharge was studied using univariate logistic regression and reporting Odds Ratios (ORs) with $95 \%$ confidence intervals $(95 \% \mathrm{CI})$. A series of gerarchical models was performed to study the association of selected variables with the presence of rehospitalization. The models studied the association of demographic variables (age and sex) alone, adding then a measure of comorbidity (CIRS severity index) or clinical conditions (AEs during index hospitalization/previous hospitalization) and them together. The last model included previous selected variables, with severity of comorbidity replaced by presence of at least moderate vascular or liver disease (the two classes of diseases significantly associated with readmission in the univariate analysis). Selection of variables to be included in the multivariate model was based on statistical and clinical significance. All statistical calculations were performed with the software JMP Pro 10 (SAS Institute Inc.).

\section{Results}

Of the 1380 enrolled patients, a total of 1178 (85\%) were discharged alive from the participating wards. The 3 month follow-up information was obtained from 832 (70\%) of them. During the 3 months of follow-up, 66 (7.9\%) patients died, thus 766 patients were ultimately included in the analysis: 145 of them (19\%) were readmitted at least once within 3 months from the index date of discharge (rehospitalized group), the remaining 621 patients were the non-rehospitalized group (Fig. 1). Among the rehospitalized group, $63(43.4 \%)$ patients were readmitted within 1 month (18 of them in the first group). Of the 766 elderly followed up females were $55.2 \%$ and $51.5 \%$ in the rehospitalized and non-rehospitalized groups, and the mean age was $77.8( \pm 7.6)$ and $78.7( \pm 7.2)$ years respectively. The mean length of hospital stay was $11.4( \pm 8.9)$ and $10.2( \pm 7.9)$ days for rehospitalized and non- rehospitalized groups.

Table 1 shows the socio-demographic and clinical characteristics of rehospitalized and non-rehospitalized groups and factors associated with readmission. By univariate analysis (Table 1), the occurrence of AEs during hospitalization, any previous hospital admission within 6 months before the index hospitalization, number of diagnosis, number of drugs, CIRS severity index, CIRS comorbidity index, and vascular and liver diseases with a level of impairment at discharge of 3 or more at CIRS were significantly associated with an increased risk of readmission, while the length of hospital stay was not. Among AEs, diagnoses included in the chapter 9 of ICD-9 (digestive system diseases) $(\mathrm{OR}=3.2,95 \% \mathrm{CI}=1.49-6.87 ; p=0.004)$ and those in the chapter 17 (injury and poisoning diseases) (OR $=5.3,95 \% \mathrm{CI}=1.60-17.67$; $p=0.008)$ were significantly associated to readmission. In the multivariate logistic regression analyses (Table 2), after adjustment for factors which were statistically significantly associated at univariate analysis, only AEs during hospitalization, previous hospital admission, and vascular and liver diseases were still significantly associated with the risk of

1380 enrolled patients

$$
\begin{aligned}
& -120 \text { transferred to another ward } \\
& -50 \text { dead } \\
& -32 \text { excluded for lacking information }
\end{aligned}
$$

1178 patients discharged alive

$$
346 \text { without follow-up information }
$$

832 patients with follow-up information 66 dead during 3 months of follow-up 766 included patients

$$
145
$$

patients readmitted whithin 3 months (rehospitalized group)
621

patients non-readmitted whithin 3 months (non-rehospitalized group)
Fig. 1. Flowchart of the study. 
Table 1

Socio-demographic and clinical characteristics of rehospitalized and not rehospitalized groups and factors associated with readmission in the univariate analysis.

\begin{tabular}{|c|c|c|c|c|c|}
\hline \multirow{2}{*}{\multicolumn{2}{|c|}{ Variables }} & \multicolumn{4}{|c|}{ Re-hospitalization } \\
\hline & & Yes $\mathrm{n}=145$ & No $\mathrm{n}=621$ & OR $(95 \% \mathrm{CI})$ & P-value \\
\hline \multicolumn{2}{|l|}{ Females, n (\%) } & $80(55.17)$ & $320(51.53)$ & $1.16(0.80-1.66)$ & 0.42 \\
\hline \multicolumn{2}{|l|}{ Age (years), mean (SD) } & $77.83(7.58)$ & $78.70(7.22)$ & $0.98(0.96-1.00)$ & 0.21 \\
\hline \multirow[t]{2}{*}{ Age groups } & $<85$ years & $116(80.00)$ & $497(80.03)$ & $1.00(0.62-1.56)$ & 0.99 \\
\hline & $>=85$ years & $29(20.00)$ & $124(19.97)$ & & \\
\hline \multicolumn{2}{|l|}{ Education (years), mean (SD) } & $7.13(4.24)$ & $7.23(4.05)$ & $0.99(0.37-2.07)$ & 0.80 \\
\hline \multirow[t]{3}{*}{ Marital status, ${ }^{a} \mathrm{n}(\%)$} & Single (unmarried, divorced, separated) & $20(13.89)$ & $54(8.71)$ & 1 & 0.13 \\
\hline & Married & $69(47.92)$ & $340(54.84)$ & $0.54(0.31-0.99)$ & \\
\hline & Widow/er & $55(38.19)$ & $226(36.45)$ & $0.65(0.36-1.21)$ & \\
\hline \multirow[t]{5}{*}{ Living arrangement, ${ }^{\mathrm{b}} \mathrm{n}(\%)$} & Alone & $29(21.01)$ & $154(25.08)$ & 1 & 0.26 \\
\hline & With spouse & $55(39.86)$ & $263(42.83)$ & $1.11(0.68-1.83)$ & \\
\hline & With sons & $27(19.57)$ & $86(14.01)$ & $1.66(0.92-3.00)$ & \\
\hline & With spouse and sons & $11(7.97)$ & $62(10.10)$ & $0.94(0.42-2.00)$ & \\
\hline & Other & $16(11.59)$ & $49(7.98)$ & $1.73(0.86-3.43)$ & \\
\hline \multicolumn{2}{|l|}{ Caregiver, ${ }^{\mathrm{c}} \mathrm{n}(\%)$} & $81(55.86)$ & $324(52.43)$ & $1.15(0.80-1.65)$ & 0.46 \\
\hline \multicolumn{2}{|c|}{ BMI (Body max Index), mean (SD) } & $26.59(5.28)$ & $26.42(5.17)$ & $1.00(0.97-1.04)$ & 0.72 \\
\hline \multicolumn{2}{|c|}{ Adverse clinical events during hospitalization, n (\%) } & $64(44.14)$ & $184(29.63)$ & $1.87(1.30-2.72)$ & 0.001 \\
\hline \multicolumn{2}{|c|}{ Previous hospital admission ( 6 months before index admission), $\mathrm{n}(\%)$} & $64(44.14)$ & $170(27.38)$ & $2.09(1.44-3.03)$ & $<0.0001$ \\
\hline \multirow[t]{2}{*}{ Risk factors } & Smoker & $16(11.03)$ & $52(8.37)$ & $1.36(0.75-2.45)$ & 0.32 \\
\hline & Drinker $^{\mathrm{d}}$ & $72(49.66)$ & $268(43.23)$ & $1.29(0.90-1.86)$ & 0.16 \\
\hline \multicolumn{2}{|c|}{ Number of diagnoses at discharge, mean (SD) } & $7.04(3.13)$ & $6.49(2.85)$ & $1.06(1.00-1.13)$ & 0.05 \\
\hline \multicolumn{2}{|c|}{ Number of drugs at discharge, mean (SD) } & $6.95(2.86)$ & $6.10(2.81)$ & $1.10(1.04-1.18)$ & 0.002 \\
\hline \multicolumn{2}{|c|}{ CIRS-severity index at discharge, mean (SD) } & $1.74(0.33)$ & $1.67(0.30)$ & $2.17(1.21-3.88)$ & 0.013 \\
\hline \multicolumn{2}{|c|}{ CIRS-comorbidity index at discharge, mean (SD) } & $3.32(1.85)$ & $2.9(1.79)$ & $1.13(1.02-1.25)$ & 0.018 \\
\hline \multicolumn{2}{|c|}{ Barthel index at admission, mean (SD) } & $79.18(28.05)$ & $82.50(26.26)$ & $0.99(0.99-1.00)$ & 0.20 \\
\hline \multicolumn{2}{|c|}{ Short Blessed Test at admission, mean (SD) } & $9.97(8.24)$ & $9.08(7.66)$ & $1.01(0.99-1.03)$ & 0.24 \\
\hline \multicolumn{2}{|c|}{ Geriatric Depression Scale at admission, mean (SD) } & $1.39(1.24)$ & $1.36(1.20)$ & $1.02(0.88-1.19)$ & 0.78 \\
\hline \multicolumn{2}{|c|}{ Duration of hospital stay, mean (SD) } & $11.39(8.89)$ & $10.15(7.86)$ & $1.07(1.00-1.04)$ & 0.12 \\
\hline \multicolumn{6}{|c|}{ Cumulative Illness Rating Scale (CIRS) at discharge-level of impairment $>=3$} \\
\hline \multicolumn{2}{|c|}{ Cardiac, n (\%) } & $71(48.97)$ & $291(46.86)$ & $1.09(0.76-1.56)$ & 0.65 \\
\hline \multicolumn{2}{|l|}{ Hypertension, n (\%) } & $70(48.28)$ & $297(47.83)$ & $1.02(0.71-1.46)$ & 0.92 \\
\hline Vascular, n (\%) & & $58(40.00)$ & 185 (29.79) & $1.57(1.08-2.28)$ & 0.02 \\
\hline Respiratory, n (\%) & & $59(40.69)$ & $243(39.13)$ & $1.06(0.74-1.54)$ & 0.73 \\
\hline Eye/ear/nose/throat, n (\%) & & $19(13.10)$ & $60(9.68)$ & $1.40(0.81-2.44)$ & 0.24 \\
\hline Upper gastrointestinal, n (\%) & & $25(17.24)$ & $116(18.68)$ & $0.91(0.56-1.46)$ & 0.69 \\
\hline Gastrointestinal, n (\%) & & $13(8.97)$ & $58(9.34)$ & $0.96(0.51-1.80)$ & 0.89 \\
\hline Liver, n (\%) & & $32(22.07)$ & $64(10.31)$ & $2.46(1.54-3.94)$ & 0.0003 \\
\hline Renal, n (\%) & & $23(15.86)$ & $81(13.04)$ & $1.26(0.76-2.08)$ & 0.38 \\
\hline Other genitourinary, n (\%) & & $23(15.86)$ & $79(12.72)$ & $1.29(0.78-2.14)$ & 0.33 \\
\hline Musculoskeletal, n (\%) & & $33(22.76)$ & $135(21.74)$ & $1.06(0.69-1.63)$ & 0.79 \\
\hline Neurological, n (\%) & & $27(18.62)$ & $107(17.23)$ & $1.09(0.69-1.75)$ & 0.69 \\
\hline Endocrine/metabolic, n (\%) & & $43(29.66)$ & $182(29.31)$ & $1.02(0.68-1.51)$ & 0.93 \\
\hline Psychiatric/behavioral catego & & $22(15.17)$ & 65 (10.47) & $1.53(0.90-2.56)$ & 0.12 \\
\hline
\end{tabular}

a $\mathrm{N}=764(144 / 620)$.

b $\mathrm{N}=752(138 / 614)$.

c $\mathrm{N}=763(145 / 618)$.

d $\mathrm{N}=765(145 / 620)$.

hospital readmission. We also analyzed the effect on readmission of the most common diseases at discharge and found that heart failure significantly affected 3 -month readmission $(\mathrm{OR}=1.9,95 \% \mathrm{CI}=1.18-2.97 ; p=$ 0.008 ). It was the second reason of the index hospitalization in patients with 3-month readmission, with a prevalence of $11 \%$ and was the first cause of readmission in the same group, with a prevalence of $13 \%$. Among liver diseases, cirrhosis at discharge was significantly associated with the likelihood of readmission $(\mathrm{OR}=1.8,95 \% \mathrm{CI}=1.12-3.05 ; p=$ $0.021)$. CIRS severity and CIRS comorbidity indexes were statistically significant only in model 2 (Table 2).

\section{Discussion}

In this study the rate of hospital readmission within 3 months from discharge (19\%) was slightly lower than the results of a previous study conducted in elderly patients (23\%) [12], and than those of the study of Jencks et al. (34\%) conducted in patients not selected for age in the frame of Medicare [3]. Our rate is almost equal to that of Maurer et al. (20\%), who performed a pilot study in patients recruited by internal medicine wards in Switzerland [6].
According to the literature, there are several factors that might be related to the risk of hospital readmission in elderly people, such as functional status score, illness severity, co-morbidity, polypharmacy, presenting illness and age [18]. This study showed that readmitted patients suffered of more chronic illnesses with a higher severity index, consumed more drugs, and were often hospitalized in the 6 months prior to the index hospitalization, developed an AE during index hospitalization and had vascular or liver diseases. These findings are broadly consistent with other observations, showing that previous hospital admission, morbidity/comorbidity and also functional disability and length of hospital stay were common risk factors in people aged $\geq 75$ years [9]. In some studies, the duration of hospital stay (defined as longer than the 90th percentile of hospital stays or than median or than 3 days) was associated with a higher risk of short term readmission [12,19-21], most likely indicative of the severity of the basic illness. In the present study the duration of hospitalization did not affect the rate of readmission. However, $44 \%$ of patients with at least one readmission within 3 months from discharge had had a hospital admission in the previous 6 months. Recurrent admissions have also been pointed out as a predictor of readmission [22], perhaps because in the elderly relapse of the 
Table 2

Risk factors affecting hospital readmission within 3 months after discharge in 5 multivariate logistic regression models.

\begin{tabular}{|c|c|c|c|c|c|c|c|c|c|c|}
\hline \multirow[b]{2}{*}{ Re-hospitalization } & \multicolumn{2}{|l|}{ Model 1} & \multicolumn{2}{|l|}{ Model 2} & \multicolumn{2}{|l|}{ Model 3} & \multicolumn{2}{|l|}{ Model 4} & \multicolumn{2}{|l|}{ Model 5} \\
\hline & OR (95\%CI) & $P$-value & OR (95\%CI) & $P$-value & OR (95\%CI) & $P$-value & OR (95\%CI) & $P$-value & OR (95\%CI) & $P$-value \\
\hline Sex (female) & $1.16(0.81-0.68)$ & 0.43 & $1.23(0.85-1.78)$ & 0.28 & $1.18(0.81-1.72)$ & 0.37 & $1.23(0.84-1.80)$ & 0.27 & $1.27(0.87-1.88)$ & 0.20 \\
\hline Age $(>=85)$ & $0.98(0.61-0.52)$ & 0.92 & $0.93(0.58-1.46)$ & 0.76 & $0.93(0.56-1.47)$ & 0.78 & $0.90(0.55-1.42)$ & 0.66 & $1.01(0.62-1.60)$ & 0.97 \\
\hline CIRS severity index & - & - & $2.24(1.25-4.03)$ & 0.007 & - & - & $1.80(0.98-3.31)$ & 0.06 & - & - \\
\hline $\begin{array}{l}\text { Adverse clinical events } \\
\text { during hospitalization }\end{array}$ & - & - & - & - & $1.78(1.22-2.60)$ & 0.003 & $1.76(1.21-2.58)$ & 0.003 & $1.74(1.19-2-56)$ & 0.0045 \\
\hline $\begin{array}{l}\text { Previous hospital admission } \\
\text { ( } 6 \text { months before current } \\
\text { admission) }\end{array}$ & - & - & - & - & $2.03(1.39-2.96)$ & 0.0003 & $1.93(1.31-2.83)$ & 0.009 & $1.92(1.31-2.82)$ & 0.0009 \\
\hline $\begin{array}{l}\text { Cardiovascular diseases } \\
\quad(\text { level of impairment } \geq 3)\end{array}$ & - & - & - & - & - & - & - & - & $1.48(1.00-2.17)$ & 0.0481 \\
\hline $\begin{array}{l}\text { Liver diseases (level of } \\
\quad \text { impairment } \geq 3 \text { ) }\end{array}$ & - & - & - & - & - & - & - & - & $2.32(1.42-3.77)$ & 0.0010 \\
\hline
\end{tabular}

Model 1: multivariate with sex and age.

Model 2: multivariate with sex, age and CIRS severity index.

Model 3: multivariate with sex, age, adverse clinical events during hospitalization and previous hospital admission.

Model 4: multivariate with sex, age, adverse clinical events during hospitalization, previous hospital admission and CIRS severity index.

Model 5: model 3 + vascular and hepatic illnesses.

index illness is the main reason for readmission within 3 months of discharge [22]. This hypothesis is supported by our findings that in patients readmitted at least once, heart failure was the second reason of the index hospitalization and the first cause of readmission.

The role of functional disability as predictor is controversial. In the study of Laniece et al. functional disability was relevant to explain the risk of early readmissions in a cohort of patients aged 75 and older [23]. However, in this study, functional disability as assessed by the Barthel index was not associated with readmission, in agreement with the findings of Alarcon et al., who showed that limited activities of daily living were only predictive of hospital mortality and prolonged stay [24].

We found no significant association between the likelihood of readmission and age, gender, marital status, education, living arrangement, BMI, smoke and alcohol consumption. In other studies readmission was mostly related to age, comorbidities and the type of chronic illnesses $[5,6,8,9]$, the role of age being explained by the fact that older people have more chronic diseases and a lower mean functional status [5].

Vascular diseases and particularly heart failure at discharge were associated to readmission, in agreement with previous observations on the predictive role of diseases of the circulatory system [12] and particularly of heart failure [25]. Also liver diseases were associated with readmission, in agreement with a previous study based upon 30-day readmission [7].

By calculating the severity index and comorbidity index on the basis of a level of impairment of 3 or more, a significant association was found between severity of illnesses and readmission rate, in agreement with [26] who found that patients readmitted during the first three months after discharge were sicker than controls (CIRS classes 3-4: $52.1 \%$ vs $34.1 \%$ ) and that those readmitted between the third and the sixth months had a significantly higher CIRS total score [26]. No previous studies investigated the effect of AEs on hospital readmission, but our previous study [13] found that the occurrence of AEs in hospital (defined as any acute clinical problem that newly occurred during hospitalization [27]) was the most significant predictor for the extension of hospital stay by nearly 4 days and increased sevenfold the risk of in-hospital death. In this study AEs that occurred during index hospitalization were associated with the likelihood to be readmitted, confirming that also a development of a new clinical problem could represent one of the principal reasons for readmission [22].

In conclusion, factors affecting hospital readmission in older patients are multiple and complex. Our results are important for clinical practice because they demonstrate that special clinical attention is needed when elderly patients are discharged from hospital with specific features such as adverse clinical events that had newly occurred during hospitalization, prior hospital admission (within 6 month of the index hospitalization), illness severity and vascular and liver diseases.

\subsection{Strengths and limitations}

The most important strength of the REPOSI study is the multicenter design that involved 66 internal medicine and geriatric wards through the country, resulting in a representative sample of the old Italian hospitalized population. Moreover, including the patients during four periods of four weeks (one per season) enabled balancing the effect of seasons on acute diseases leading to hospitalization. Collecting data from the patients by the physicians allowed the analysis of some variables often omitted in studies that relied on administrative database, such as severity of the illness, adverse clinical events which occurred during hospitalization, risk factors, clinical parameters, social and physical characteristics of the patients (education, marital status, living arrangement, and body mass index).

Anyway some limitations deserve to be cited. First, the 3 months of follow-up was not performed in all patients discharged, providing a lack of information and an incomplete picture of the sample. Second, the phone interviews collected from patients themselves could not be realistic or complete due to the fact that the elderly may not be always aware of their drug regimen, $\mathrm{AE}$, and so on. Third, the problem with under-reported conditions such as depression has been previously reported [28]. Fourth, we didn't collect some important information such as Barthel index at discharge or destination at discharge, that are factors that may influence the risk of hospital readmission of elderly patients. Finally, we focused our attention only on internal medicine and geriatrics wards avoiding a generalization of our observations to all in-hospital settings.

\section{Learning points}

- $19 \%$ of patients discharged from a representative sample of Italian internal medicine and geriatric wards were rehospitalized at least once within 3 months.

- There was no significant association between some sociodemographic and clinical characteristics (length of stay, cognitive, functional and depression status) and probability of readmission.

- New adverse clinical events that had occurred during index hospitalization, prior hospital admission, illness severity and vascular and liver diseases with level of impairment $\geq 3$ (according to Cumulative Illness Rating Scale-CIRS) were the main predictors of a later rehospitalization after discharge. 
- Among cardiovascular and liver diseases, heart failure and cirrhosis were the most frequent diseases of the two categories.

- The identification of these factors leading to hospital readmission should prompt a more accurate surveillance of these patients in the attempt to reduce this adverse outcome.

\section{Conflict of interests}

All authors have no conflicts of interests to disclose.

\section{Appendix I. Members of the REPOSI (REgistro POliterapie Società Italiana medicina interna) Study}

Steering Committee: Pier Mannuccio Mannucci (Chair, Fondazione IRCCS Cà Granda Ospedale Maggiore Policlinico, Milano), Alessandro Nobili (co-chair, Istituto di Ricerche Farmacologiche "Mario Negri", Milano), Mauro Tettamanti, Luca Pasina, Carlotta Franchi (Istituto di Ricerche Farmacologiche "Mario Negri", Milano), Francesco Salerno (IRCCS Policlinico San Donato Milanese, Milano), Salvatore Corrao (Azienda Ospedaliera Universitaria Policlinico P. Giaccone di Palermo, Palermo), Alessandra Marengoni (Spedali Civili di Brescia, Brescia), Alfonso Iorio (McMaster University, Hamilton, Canada), Maura Marcucci (Azienda Ospedaliera Santa Maria della Misericordia, Perugia).

Clinical data monitoring and revision: Valentina Spirito, Damia Noce, Jacopo Bonazzi, Rossana Lombardo, Eleonora Sparacio, Stefania Alborghetti (Istituto di Ricerche Farmacologiche "Mario Negri", Milano).

Database Management and Statistics: Mauro Tettamanti, Luigi De Vittorio, Codjo Djignefa Djade (Istituto di Ricerche Farmacologiche "Mario Negri", Milano).

Investigators: Giuseppe Paolisso, Maria Rosaria Rizzo, Maria Teresa Laieta (Azienda Ospedaliera Universitaria della Seconda Università degli Studi di Napoli, Napoli, VI Divisione di Medicina Interna e Malattie Nutrizionali dell'Invecchiamento); Torella Roberto, Marcello Persico, Teresa Salvatore, Ferdinando Carlo Sasso (Azienda Ospedaliera Universitaria della Seconda Università degli Studi di Napoli, Napoli, Medicina Interna e Malattie Epato-Bilio Metaboliche Avanzate); Riccardo Utili, Emanuele Durante Mangoni, Daniela Pinto (Azienda Ospedaliera Universitaria della Seconda Università degli Studi di Napoli, Napoli, Medicina Infettivologica e dei trapianti); Luigi Fenoglio, Chiara Brignone, Christian Bracco (Azienda Sanitaria Ospedaliera Santa Croce e Carle di Cuneo, Cuneo, Medicina Interna); Laura Gasbarrone, Patrizia Porcari, Giseppe Famularo, Maria Rosaria Sajeva (Azienda Ospedaliera Ospedale San Camillo, Roma, Medicina Interna 1); Giorgio Maniscalco, Massimo Gunelli, Daniela Tirotta (Azienda Ospedaliera Ospedale San Salvatore, Pesaro, Soc Medicina Interna); Giuseppe Realdi, Aldo Baritussio, Francesco Frassoni (Azienda Ospedaliera Università di Padova, Padova, Clinica Medica I); Roberto Delsignore, Maria Cristina Baroni, Marianna Zardo, Azienda Ospedaliera Università di Parma, Parma, Clinica e Terapia Medica Renato Fellin , Stefano Volpato , Sioulis Fotini (Azienda Ospedaliera Universitaria Ospedale Sant'Anna, Ferrara, Unità Operativa di Medicina Interna Gerontologia e Geriatria); Roberto Manfredini, Carlo Longhini, Christian Molino, Elena Incasa, (Azienda Ospedaliera Università Ospedale Sant'Anna, Ferrara, Unità Operativa Clinica Medica); Gianfranco Guarnieri, Michela Zanetti, Annalisa Spalluti (Azienda Ospedaliera Universitaria Ospedali Riuniti di Trieste, Trieste, Clinica Medica Generale e Terapia Medica); Giovanbattista Rini, Pasquale Mansueto, Ilenia Pepe (Azienda Ospedaliera Universitaria Policlinico P. Giaccone di Palermo, Palermo,
Medicina Interna e Malattie Metaboliche); Giuseppe Licata, Luigi Calvo, Maria Valenti; Antonino Tuttolomondo; Riccardo Di Sciacca (Azienda Ospedaliera Universitaria Policlinico P. Giaccone di Palermo, Palermo, Medicina Interna e Cardioangiologia); Salvatore Antonaci, Francesco Vella, Alessandro Marseglia (Azienda OspedalieroUniversitaria Consorziale Policlinico di Bari, Bari, Clinica Medica II Frugoni); Vincenzo Centonze, Maria Ester Modeo (Azienda Ospedaliero-Universitaria Consorziale Policlinico di Bari, Bari, Medicina Interna Ospedale "Pende-Ferrannini"); Giuseppe Palasciano, Stefania Pugliese, Caterina Capobianco (Azienda Ospedaliero-Universitaria Consorziale Policlinico di Bari, Bari, Medicina Interna Murri); Giovanni Murialdo, Marta Bovio (IRCS Azienda Ospedaliera Universitaria San Martino-IST di Genova, Genova, Clinica di Medicina Interna 2); Franco Laghi Pasini, Pier Leopoldo Capecchi, Maurizio Bicchi (Azienda Ospedaliera Universitaria Senese, Siena, Unità Operativa Complessa Medicina 2); Ranuccio Nuti, Roberto Valenti, Cosimo Capodarca (Azienda Ospedaliera Università Senese, Siena, Medicina Interna I); Alberto Auteri, Anna Laura Pasqui, Luca Puccetti (Azienda Ospedaliera Universitaria Senese, Siena, Medicina 3); Oliviero Olivieri, Anna Maria Stanzial (Azienda Ospedaliera Universitaria Integrata di Verona, Verona, Unità Operativa di Medicina Interna B); Giancarlo Agnelli, Alfonso Iorio, Maura Marcucci, Ana Macura (Azienda Ospedaliera Santa Maria della Misericordia, Perugia, Medicina Interna e Cardiovascolare); Elmo Mannarino, Graziana Lupattelli, Pamela Rondelli (Azienda Ospedaliera Santa Maria della Misericordia, Perugia, Medicina Interna, Angiologia, Malattie da Arteriosclerosi); Maria Grazia Serra (Azienda Ospedaliera "Cardinale Panico" di Tricase, Lecce, Unità Operativa Complessa Medicina); Giuseppe Musca, Olga Cuccurullo (Azienda Sanitaria Provinciale di Cosenza Presisio Ospedaliero di Cetraro, Cosenza, Unità Operativa Complessa Medicina Interna); Maria Domenica Cappellini, Giovanna Fabio, Irene Motta, Flavio Cantoni (Fondazione IRCCS Cà Granda Ospedale Maggiore Policlinico, Milano, Unità Operativa Medicina Interna IA); Silvia Fargion, Paola Bonara, Mara Bulgheroni (Fondazione IRCCS Cà Granda Ospedale Maggiore Policlinico, Milano, Medicina Interna 1B); Fabio Magrini, Ferdinando Massari, Tatiana Tonella (Fondazione IRCCS Cà Granda Ospedale Maggiore Policlinico, Milano, Unità Operativa Medicina Cardiovascolare); Flora Peyvandi, Alberto Tedeschi, Raffaella Rossio (Fondazione IRCCS Cà Granda Ospedale Maggiore Policlinico, Milano, Medicina Interna 2); Guido Moreo, Barbara Ferrari (Fondazione IRCCS Cà Granda Ospedale Maggiore Policlinico, Milano, Medicina Interna 3); Valter Monzani, Valeria Savojardo, Christian Folli (Fondazione IRCCS Cà Granda Ospedale Maggiore Policlinico, Milano, Medicina d'Urgenza); Daniela Mari, Paolo Dionigi Rossi, Eleonora Ziglioli (Fondazione IRCCS Cà Granda Ospedale Maggiore Policlinico, Milano, Geriatria); Maria Sole Lilleri (Fondazione IRCCS Cà Granda Ospedale Maggiore Policlinico, Milano, Medicina Generale ad Indirizzo Geriatrico); Carlo Selmi, Francesca Meda (IRCCS Istituto Clinico Humanitas, Milano, Clinica Medica); Francesco Salerno, Massimo Cazzaniga, Valentina Monti (IRCCS Policlinico San Donato Milanese, Milano, Medicina Interna I); Carlo L. Balduini, Giampiera Bertolino, Luca Dezzani, Piergiorgio Cavallo (IRCCS Policlinico San Matteo di Pavia, Pavia, Clinica Medica III); Gino Roberto Corazza, Emanuela Miceli (IRCCS Policlinico San Matteo di Pavia, Pavia, Clinica Medica I, Reparto 11); Maria Beatrice Secchi, Sheng Chin Wu, (Ospedale Bassini di Cinisello Balsamo, Milano, Divisione Medicina); Luigi Anastasio, Lucia Sofia, Maria Carbone, Leonardo Bertucci (Ospedale Civile Jazzolino di Vibo Valentia, Vibo Valentia, Medicina Generale); Giancarlo Traisci, Lucrezia De Feudis (Ospedale Civile Santo Spirito di Pescara, Pescara, Medicina Interna); Elisabetta 
Bergami, Emanuela Rizzioli (Ospedale del Delta, Lagosanto, Ferrara, Medicina Interna); Carlo Cagnoni, Luca Bertone, Antonio Manucra (Ospedale di Bobbio, Piacenza, Unità Operativa Medicina e Primo Soccorso); Esio Ronchi, Alberto Buratti, Tiziana Tognin, Daniele Bertolini, Nicola Lucio Liberato (Ospedale di Casorate Primo Carlo Mira, Pavia, Unità Operativa Medicina Generale); Giordano Bernasconi, Barbara Nardo (Ospedale di Circolo di Busto Arsizio, Varese, Medicina I); Achille Venco, Luigina Guasti, Lorenzo Maroni, Luana Castiglioni (Università degli Studi dell'Insubria, Ospedale di Circolo e Fond Macchi Varese, Medicina Interna I); Massimo Vanoli, Giulia Grignani, Gianluca Casella (Azienda Ospedaliera della Provincia di Lecco Ospedale di Merate, Lecco, Medicina Interna); Gerardo Mancuso, Rosa Tavella, Rosaria Persico (Ospedale Giovanni Paolo II Lamezia Terme, (CZ), Unità Operativa Complessa Medicina Interna); Marco Cicardi, Giulia Sandrone (Ospedale Luigi Sacco, Milano, Medicina II); Marina Magenta, Francesca Perego; Maria Rachele Meroni (Ospedale Luigi Sacco, Milano, Medicina $3^{\circ}$ ); Renzo Rozzini, Lina Falanga, Alessandro Giordano (Ospedale Poliambulanza, Brescia, Medicina Interna e Geriatria); Giorgio Menardo, Stefania Bottone, Elsa Sferrazzo (Ospedale San Paolo, Savona, Medicina Interna e Gastroenterologia); Rodolfo Tassara, Deborah Melis, Lara Rebella (Ospedale San Paolo, Savona, Medicina I); Claudio Ferri, Rinaldo Striuli, Rosa Scipioni (Ospedale San Salvatore, L'Aquila, Medicina Interna Universitaria); Raffaella Salmi, Piergiorgio Gaudenzi, Filippo Di Todaro, Ingrid Nielsen, Lisa Giusto (Ospedale Sant'Anna, Ferrara, Medicina Alta Rotazione); Andrea Semplicini, Lucia Gottardo (Ospedale SS. Giovanni e Paolo, Venezia, Medicina Interna 1); Giuseppe Delitala, Stefano Carta, Sebastiana Atzori (Ospedale Universitario Policlinico di Sassari, Sassari, Clinica Medica); Enrico Agabiti Rosei, Damiano Rizzoni, Luana Castoldi (Spedali Civili di Brescia, Brescia, Seconda Medicina); Emanuele Altomare, Gaetano Serviddio, Santina Salvatore (Ospedali Riuniti di Foggia, Foggia, Medicina Interna Universitaria II); Giacomo Fera, Maria Loreta Di Luca, Donatella Renna (Ospedale San Giacomo di Monopoli, Bari, Unità Operativa Medicina Interna); Antonio Picardi, Chiara Mazzarelli, Umberto Vespasiani Gentilucci, Antonio De Vincentis, Dritan Hila (Università Campus Bio-Medico, Roma, Medicina Clinica e Patologia); Mauro Bernardi, Silvia Li Bassi, Luca Santi (Azienda Ospedaliera Policlinico Sant'Orsola-Malpighi, Bologna, Semeiotica Medica Bernardi); Cesare Masala, Antonio Mammarella, Valeria Raparelli (Policlinico Umberto I, Roma, Medicina Interna D); Filippo Rossi Fanelli, Massimo Delfino, Antonio Amoroso (Policlinico Umberto I, Roma, Medicina Interna $\mathrm{H}$ ); Pietro Serra, Vincenzo Fontana, Marco Falcone (Policlinico Umberto I, Roma, Terza Clinica Medica); Francesco Violi, Stefania Basili, Ludovica Perri (Policlinico Umberto I, Roma, Prima clinica Medica); Nicolò Gentiloni Silveri, Guido De Marco, Bianca Giupponi (Policlinico Universitario A. Gemelli, Roma, Unità Operativa Complessa Medicina d'Urgenza e Pronto Soccorso); Raffaele Landolfi, Antonio Grieco, Antonella Gallo (Policlinico Universitario A. Gemelli, Roma, Clinica Medica); Francesco Perticone, Angela Sciacqua, Michele Quero, Chiara Bagnato (Università Magna Grecia Policlinico Mater Domini, Catanzaro, Unità Operativa Malattie Cardiovascolari Geriatriche); Paola Loria, Stefano Ballestri, Maria Angela Becchi (Università Studi di Modena e Reggio Emilia, OCSAE Baggiovara, Modena, Medicina Interna Indirizzo Metabolico Nutrizionistico); Luigi Bolondi, Leonardo Rasciti, Ilaria Serio, Silvia Gualandi (Policlinico Sant'Orsola-Malpighi, Bologna, Unità Operativa Complessa Medicina Interna); Giuseppe Romanelli, Alessandra Marengoni, Francesca Bonometti (Spedali Civili di Brescia, Brescia, Geriatria); Nicola Carulli, Stefania Rondinella, Iolanda Giannico (Medicina Metabolica, Università di Modena e Reggio Emilia); Franco Dallegri, Luciano Ottonello, Alessandra Quercioli, Alessandra Barreca (Medicina Interna 1,
Università di Genova); Andrea Sacco, Antonio Bonelli, Gaetano Dentamaro (Medicina, Ospedale Madonna delle Grazie, Matera); Giuliana Micale (Medicina Generale ad indirizzo Geriatrico, IRCCS Istituto Auxologico Italiano, Milano); Giuseppe Delitalia, Silvia Deidda, Luciana Maria Cuccuru (Clinica Medica, Azienda Mista Ospedaliera Universitaria, Sassari); Giampiero Benetti, Michela Quagliolo, Giuseppe Riccardo Centenaro (Medicina 1, Ospedale di Melegnano, Vizzolo Predabissi, Milano); Antonio Perciccante, Alessia Coralli (Medicina, Ospedale San Giovanni-Decollato-Andisilla, Civita Castellana); Cosimo Morabito, Roberto Fava (Medicina, Ospedale Scillesi d'America, Scilla); Luisa Macchini, Anna Realdi (Clinica Medica 4, Università di Padova); Luigi Cricco, Alessandra Fiorentini, Cristina Tofi (Geriatria, Ospedale di Montefiascone).

\section{References}

[1] Alonso Martínez JL, Llorente Diez B, Echegaray Agara M, Urbieta Echezarreta MA González Arencibia C. Hospital readmission in internal medicine. An Med Interna 2001;18:248-54.

[2] Barba Martin R, Marco Martinez J, Plaza Canteeli S, Gomez Rodrigo J, de la Cervero Jimenez M, Solis Villa J. Retrospective study of early readmissions at an internal medicine service. Rev Clin Esp 2000;200:252-6.

[3] Jencks SF, Williams MV, Coleman EA. Rehospitalizations among patients in the Medicare Fee-for-Service Program. N Engl J Med 2009;360:1418-28.

[4] Coleman EA, Parry C, Chalmers S, Min S. The Care Transitions Intervention. Results of a randomized controlled trial. Arch Intern Med 2006;166:1822-8.

[5] Zamir D, Zamir M, Reitblat T, Zeev W, Polishchuk I. Readmissions to hospital within 30 days of discharge from the internal medicine wards in southern Israel. Eur J Intern Med 2006;17:20-3.

[6] Maurer PP, Ballmer PE. Hospital readmissions-are they predictable and avoidable? Swiss Med Wkly 2004;134:606-11.

[7] Silverstein MD, Qin H, Mercer SQ, Fong J, Haydar Z. Risk factors for 30-day hospital readmission in patients $\geq 65$ years of age. Proc (Bayl Univ Med Cent) 2008;21: 363-72.

[8] Kansagara D, Englander H, Salanitro A, Kagen D, Theobald C, Freeman M, et al. Risk prediction models for hospital readmission: a systematic review. JAMA 2011;306: 1688-98.

[9] Garcia-Perez L, Linertova R, Lorenzo-Riera A, Vazquez-Diaz JR, Duque-Gonzalez B, Sarria-Santamera A. Risk factors for hospital readmissions in elderly patients: a systematic review. QJM 2011;104:639-51.

[10] Kalra AD, Fisher RS, Axelrod P. Decreased length of stay and cumulative hospitalized days despite increased patient admissions and readmissions in an area of urban poverty. J Gen Intern Med 2010;25:930-5.

[11] Jones J. Readmission rates: the price of early discharge. Health Serv J 1986;19:825

[12] Cornette P, D'Hoore W, Malhomme B, Van Pee D, Meert P, Swine C. Differential risk factors for early and later hospital readmission of older patients. Aging Clin Exp Res 2004;17:322-8

[13] Nobili A, Licata G, Salerno F, Pasina L, Tettamanti M, Franchi C, et al. Polypharmacy length of hospital stay, and in-hospital mortality among elderly patients in internal medicine wards. The REPOSI study. Eur J Clin Pharmacol 2011;67:507-19.

[14] Parmelee PA, Thuras PD, Katz IR, Lawton MP. Validation of a measure of physical burden at autopsy: the cumulative illness rating scale. J Am Geriatr Soc 1995;43: 130-7.

[15] Shah S, Vanclay F, Cooper B. Improving the sensitivity of the Barthel index for stroke rehabilitation. J Clin Epidemiol 1989;42:703-9.

[16] Katzman R, Brown T, Fuld P, Peck A, Schechter R, Schimmel H. Validation of a Short Orientation-Memory-Concentration Test of cognitive impairment. Am J Psychiatry 1983;140:734-9.

[17] Hickie C, Snowdon J. Depression scales for the elderly: GDS, Gilleard, Zung. Clin Gerontol 1987;6:51-3.

[18] Campbell SE, Seymour DG, Primrose WR. A systematic literature review of factors affecting outcome in older medical patients admitted to hospital. Age Ageing 2004;33:110-5.

[19] Zanocchi M, Maero B, Martinelli E, Cerrato F, Corsinovi L, Gonella M, et al. Early re-hospitalization of elderly people discharged from a geriatric ward. Aging Clin Exp Res 2006;18:63-9.

[20] Zapatero A, Barba R, Marco J, Hinojosa J, Plaza S, Losa JE, et al. Predictive model of readmission to internal medicine wards. Eur J Intern Med 2012;23:451-6.

[21] Bisharat N, Handler C, Schwartz N. Readmissions to medical wards: analysis of demographic and socio-medical factors. Eur J Intern Med 2012;23:457-60.

[22] Williams EI, Fitton F. Factors affecting early unplanned readmission of elderly patients to hospital. BMJ 1988;297:784-7.

[23] Lanièce I, Couturier P, Dramé M, Gavazzi G, Lehman S, Jolly D, et al. Incidence and main factors associated with early unplanned hospital readmission among French medical inpatients aged 75 and over admitted through emergency units. Age Ageing 2008;3:416-22.

[24] Alarcón T, Bárcena A, González-Montalvo JI, Penãlosa C, Salgado A. Factors predictive of outcome on admission to an acute geriatric ward. Age Ageing $1999 ; 28: 429-32$. 
[25] Tavazzi L, Maggioni AP, Lucci D, Cacciatore G, Ansalone G, Oliva F, et al. Nationwide survey on acute heart failure in cardiology ward services in Italy. Eur Heart J 2006;27:1207-15.

[26] Di Iorio A, Longo AL, Mitidieri Costanza A, Bandinelli S, Capasso S, Gigante M, et al. Characteristics of geriatric patients related to early and late readmissions to hospital. Aging (Milano) 1998;10:339-46.
[27] Bernardini B, Meinecke C, Zaccarini C, Bongiorni N, Fabbrini S, Gilardi C, et al. Adverse clinical events in dependent long-term nursing home residents. J Am Geriatr Soc 1993;41:105-11.

[28] Marengoni A, Bianchi G, Nobili A, Tettamanti M, Pasina L, Corrao S, et al. Prevalence and characteristics of antidepressant drug prescriptions in older Italian patients. Int Psychogeriatr 2011;8:1-8. 